\title{
Intelligently managing mixed traffic flows at intersections Proposal of a platoon-based round-robin algorithm with priorities
}

This paper was downloaded from TechRxiv (https://www.techrxiv.org).

\section{LICENSE}

CC BY-NC-SA 4.0

SUBMISSION DATE / POSTED DATE

$06-01-2021 / 11-01-2021$

\section{CITATION}

Moradi, Hossein; Sasaninejad, Sara; Wittevrongel, Sabine; Walraevens, Joris (2021): Intelligently managing mixed traffic flows at intersections Proposal of a platoon-based round-robin algorithm with priorities. TechRxiv. Preprint. https://doi.org/10.36227/techrxiv.13525853.v1

$\mathrm{DOI}$ 
This work has been submitted to the IEEE for possible publication. Copyright may be transferred without notice, after which this version may no longer be accessible. 


\title{
Intelligently managing mixed traffic flows at intersections: Proposal of a platoon-based round-robin algorithm with priorities
}

\author{
Hossein Moradi ${ }^{\mathrm{a}, *}$, Sara Sasaninejad ${ }^{\mathrm{a}}$, Sabine Wittevrongel $^{\mathrm{a}}$, Joris Walraevens ${ }^{\mathrm{a}}$ \\ ${ }^{a}$ SMACS Research Group, Department of Telecommunications and Information Processing, Ghent University
}

\begin{abstract}
The growth of vehicle ownership has necessitated the adoption of new approaches to cope with the arising problems. In this regard, while technological advancement in Connected and Automated Vehicles (CAVs) leads to a new source of information upon which effective intersection control mechanisms can be built, it also necessitates considering the issue of mixed traffic (consisting of both CAVs and human drivers), on the one hand, and enhancing current infrastructures, on the other. This paper proposes a framework, which enhances pre-timed signals to incorporate CAVs and wireless communications. In this framework, Round Robin has been selected as the main algorithm treating vehicles' platoons as the units. Dealing with mixed traffic conditions and embedding an algorithm for prioritization of special vehicles are also discussed in the context of this framework. The result is a proposal of the Platoon-based Round Robin algorithm with Priorities (the PRRP-framework). This framework is further integrated into a speed advisory system to mutually address the issue of emissions. The results of simulations (1) show that the PRRP-framework is promising even with a low proportion of CAVs and (2) support the argument that moving towards practical solutions has a basic requirement: integration.
\end{abstract}

\section{Introduction}

The increasing number of vehicles in cities makes the mechanism of intersection management subject to many complexities, in many cases resulting in excessive queues, and consequently, economic, social, and environmental problems. The widespread occurrence of such consequences has led to a plethora of research studies on possible ways to manage the complexities of intersections. Whereas the complexities of conventional intersections are still being discussed among scholars, progress in wireless communications together with the presence of sensing technologies, and the advent of Connected and Automated Vehicles (CAVs) have instigated the issue of intelligently managing intersections.

Opportunities resulting from the capability of CAVs in sending and receiving information have led to the development of innovative intersection control mechanisms [1]. For example, in 2], the authors have used a reservation-based strategy to describe a new mechanism for managing intersections. In this mechanism, an intersection is divided into a grid of tiles, which are supposed to be reserved by vehicles ahead of the intersection. However, according to the experimental results, this approach cannot make any efficiency improvement to that of traditional traffic lights when a proportion of vehicles is still human-driven (even if that proportion is low). Considering this point, some frameworks have been proposed to address the challenge of managing mixed traffic by using traffic sensors [3. However, deploying traffic sensors (e.g., detectors) everywhere is practically neither cheap nor easy [4]. Furthermore, given the incremental permeation of any technology [5], it is particularly not

\footnotetext{
${ }^{*}$ Corresponding author

Email address: Hossein.Moradi@ugent.be (Hossein Moradi)
} 
a promising approach (at least in the near future) to look at the issue of intelligently managing intersections from a fully connected environment standpoint. Accordingly, it is essential to look for an admissible way to use the information of CAVs for effectively managing intersections of the near future without resorting to the assumption of a fully connected environment.

To effectively manage intersections, we should address the issue of demand fluctuations. It is unrealistic to assume that traffic demand remains equal during any period 6]. Moreover, traffic demands in the approaching links of an intersection might be different. Therefore, an appropriate strategy should be adopted to (1) consider the congestion state of each approaching link and (2) determine the amount of service each link receives under its congestion state in order to prevent excessive congestion and backward propagation of traffic.

Furthermore, it would also be interesting to implement a priority scheme for emergency vehicles, on the one hand, and to reduce stop-and-go events of heavy vehicles (buses, trucks, etc.) and the accompanying emissions [7], on the other. In this regard, some studies have focused on emergency vehicle preemption [8], transit signal priority [9, 10, or considering different priority treatments for different classes of vehicles simultaneously [11. It is important to note, however, the more comprehensive the priority algorithm, the more complex the intersection control mechanism becomes. Therefore, there should be a trade-off between comprehensiveness and complexity to reach a practical solution.

Concerning the issue of complexity, caution should be taken to deal with the challenges resulting from the analysis of a high volume of data. As a case in point, in [12], a modified solution-tree-generation algorithm based on a permutation order has been applied to obviate the invalid orders of driving and eliminate the unsafe patterns of sequencing. Subsequently, the virtual vehicle mapping technique has also been used to guarantee safety in the merging lanes. In another case, in [13], the authors have looked into the issue of intelligently managing intersections from the machine scheduling perspective and present an objective function to minimize the total delay at an intersection by considering mixed traffic. In this regard, a new phase-time-traffic hyper network model has been developed to incorporate the interaction among traffic signal operations and heterogeneous traffic. While using such an optimization-based approach has led to remarkable results in terms of different measures of efficiency, the obtained models cannot usually be used for real-time intersection management due to the computation intensity.

While intelligently managing intersections with considering complexities including the issues of mixed traffic fleets, demand fluctuations, priorities, and computational complexity seems to be a promising research topic (due to the current traffic condition and also the future perspective), to the best of the authors' knowledge, there is a lack of studies looking at the full picture. Accordingly, this research contributes to the literature by developing a rule-based framework that results from enhancing the current infrastructures (pre-timed signals, in particular, due to their extent in worldwide traffic networks) to efficiently manage mixed traffic at intersections while also considering the other mentioned complexities.

To this end, this study employs the Round Robin algorithm $(R R)$ as the main algorithm. This decision has been made by reviewing the properties of Fair Queueing Schedulers previously used in telecommunication networks (for detailed information see [14, 15] and the corresponding references) and is driven by the comparative advantages of the $R R$ algorithm over others in simplicity and alignment with the operation of current signals. These key attributes make this algorithm suitable for managing mixed traffic. Within this algorithm, we also discuss how best to deal with the issue of demand fluctuations, on the one hand, and embed an algorithm for prioritization of special vehicles, on the other. This altogether results in the proposal of the Platoon-based 
Round Robin algorithm with Priorities (the PRRP-framework). We have evaluated the efficiency of the PRRPactuated mechanism by means of simulations.

The second contribution of this study is that it further enables bidirectional communication and integrates the proposed PRRP-framework into a speed advisory system to reach an integrated framework. Previous studies highlighted that improving any urban system entails strong integration among its elements [16]. An urban road network is no exception and, therefore, we have proposed a framework consisting of the PRRP-framework and a speed advisory system to (respectively) use the information of CAVs and the information of the intersection controller to (mutually) address the issue of emissions while also taking the issue of average delay into account. Finding an agreeable solution for these measures based on the emergent opportunities associated with wireless communication initiatives has remained an open topic for research projects [7, which is also aimed at by this research.

The efficiency of the integrated framework is evaluated by means of simulations in VISSIM. Since, however, some research studies have raised concerns on the accuracy of VISSIM in evaluating emissions (see [17] as an example), this micro-simulator is employed here not for measuring emissions quantitatively, but for relatively comparing the framework in a general intersection with the standard control mechanism. While alternative simulation tools like SUMO [18] are also available, VISSIM has been selected due to its comparative advantages in modeling multiple types of vehicles in different road network configurations managed by different methods.

The remainder of this paper is structured as follows. Section 2 develops the PRRP-framework. Section 3 provides the simulation results to show the operation and evaluate the efficiency of the PRRP-framework. Section 4 explains how the integration of the PRRP-framework with a speed advisory system can be undertaken to address the issues of average delay and emissions. Finally, Section 5 provides some conclusions and future research directions.

\section{The PRRP-framework}

This study considers a general type of intersection that appears in traffic networks. This intersection consists of four links, each of which has two approaching lanes ${ }^{1}$ whose trajectories are shown in Fig. 1. In each lane, there can be different types of vehicles including CAVs and conventional vehicles and different classes of vehicles including special vehicles (emergency and heavy vehicles) and ordinary vehicles.

In this intersection, we have chosen to classify compatible lanes (with the predefined trajectories) consistent with the standard NEMA (National Electrical Manufacturers Association) structure, as is marked in Fig. 1 . Based on this structure, straight and left-turning lanes (which are labeled by even and odd numbers, respectively) form two sets, each of which consists of two rings. Using these sets and rings, the compatible lanes can be identified (e.g., lanes 1 and 6 or with lanes 1 and 5) and determined for the phase allocations at signalized intersections. It is worth mentioning here that the classification of compatible lanes can follow other patterns too. For example, we could also consider lanes 3 and 6 as a group of compatible lanes. However, it is important to have a sequencing paradigm, which can (1) be assimilated with current infrastructures and (2) be easily complied with by human drivers.

\footnotetext{
${ }^{1}$ We have excluded the right-turning lanes from the process since the complexities of managing an intersection are related to the lanes that need to be sequenced.
} 


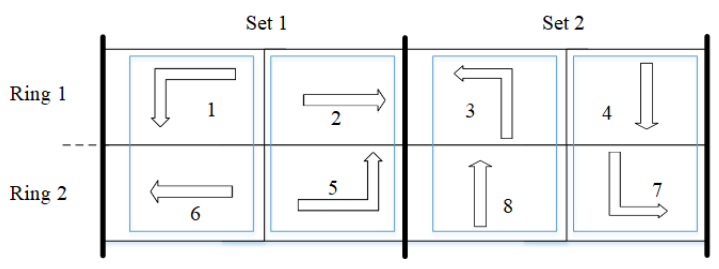

Figure 1: Standard NEMA structure

Finally, as mentioned in Section 1, we have chosen to apply RR to distribute right-of-way among identified groups of compatible lanes. In a telecommunication context, this algorithm operates as is sketched in Fig. 2 and gives permission to the respective servers to transmit their corresponding data packet (if any). Now, by considering this algorithm in the context of intersections, first, we outline the PRRP-framework in Section 2.1. Then, we employ the potentials of probability theory to derive the constitutive equations to implement an example of the PRRP-framework in Section 2.2

\subsection{The outline of the PRRP-framework}

By considering the defined groups of compatible lanes as the servers of RR, the first problem is the configuration of vehicles in such a way that they can be treated as data packets (the units of $R R$ ). In this regard, considering vehicles individually (as data packets) is (1) not optimal in terms of efficiency even if safety margins are small, (2) not easy for human drivers to comply with, especially when compatible lanes are served in parallel, and (3) not advantageous in required computations. Therefore, it is logical to choose a platoon of vehicles as the unit of $R R$.

A platoon is generally defined as a group of vehicles that move together with relatively short headways due to the presence of moving bottlenecks. The PRRP-framework treats platoons of vehicles as the units with the following objectives:

(1) Managing varying demands: the number of vehicles in each platoon correlates with the traffic condition (there are more vehicles in a platoon at busy times or in busy lanes).

(2) Reducing the number of stop-and-go events: serving platoons instead of individual vehicles results in the extension of green time until there is a big enough gap in a fleet.

It has been proved that with platooning there will also be potential for enhancing the overall throughput at intersections [19], reducing vehicle emissions [20, and improving computational efficiency [21]. However, before

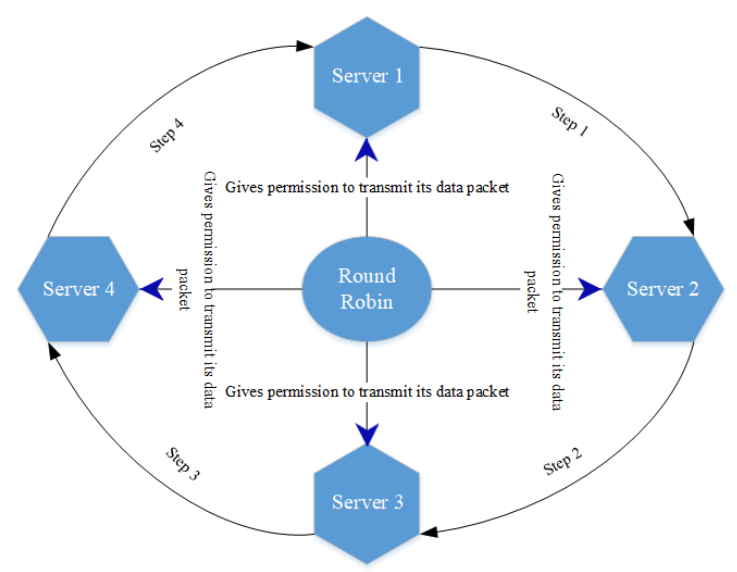

Figure 2: The operation of the RR algorithm in a telecommunication context 
getting treatment, platoons should be identified while the intersection controller neither controls nor knows the complete traffic condition at the intersection nor the approaching platoons to the intersection ${ }^{2}$, Accordingly, we use the information of CAVs to obtain an estimate of the platoon length $P L_{i}$ in group $i$ of compatible lanes. Note that the estimate $P L_{i}$ will eventually allow us to determine the required green time $G_{i}$ for group $i$ under the PRRP-framework by

$G_{i}=P L_{i} / S_{i}$

where $S_{i}$ is the service rate that can be defined based on the geometric condition of the intended intersection. Now, to approximate $P L_{i}$, we more specifically propose to use

$P L_{i} \approx E\left[N_{i} \mid I_{c}\right]+E\left[J\left(V_{i}, Z_{i}\right)\right]$

In Equation (2), the term $E\left[N_{i} \mid I_{c}\right]$ is the expected number of queued up vehicles (in group $i$ of compatible lanes) at the end of the associated red time given the information $I_{c}$ of CAVs at that moment. The second term $E\left[J\left(V_{i}, Z_{i}\right)\right]$ in Equation (2) is the estimated number of vehicles that join the queued up vehicles to cross the intersection within the same platoon. We have concentrated on these two terms, and we do so for the following reasons:

(1) Queued up vehicles (approximated by $E\left[N_{i} \mid I_{c}\right]$ in Equation (2)) maintain short headways and, therefore, we have to incorporate them in the same platoon. It is important to take this issue into account, since overflow queues (i.e., vehicles that cannot cross the intersection in the same cycle in which they arrive) highly influence the issues of average delay and emissions.

(2) Newly arriving vehicles (with relatively large headways) can still join the queued up vehicles into one platoon due to the plausible difference between the speed of queue dissipation and newly arriving vehicles. Considering this issue plays an important role in reducing the number of stop-and-go events. Accordingly, given $T_{t}^{3}$ seconds as the maximum headway in a platoon, if we define $V_{i}$ as the total number of arrivals in group $i$ during the time $g_{i}$ that is needed for the crossing of the queued up vehicles $\left(E\left[N_{i} \mid I_{c}\right]\right)$, we also need to take $Z_{i}$ into account which is the number of vehicles (from $V_{i}$ ) that move with headways shorter than $T_{t}$ and join the queued up vehicles until a headway exceeds $T_{t}$ for the first time. We have shown the expected number of vehicles that results from this interaction by $E\left[J\left(V_{i}, Z_{i}\right)\right]$ in Equation (2).

It should be noted here that in case of heavy traffic in a group of compatible lanes, the associated platoon length $\left(P L_{i}\right)$ will become very large and, therefore, other groups cannot get enough services. For this reason, we assume a limitation of $X_{t}^{4}$ vehicles on the maximum platoon size.

We shall further prepare the intersection controller for different treatments needed for effectively managing different types and classes of vehicles in each link by adding priorities for special vehicles to the picture. We can do this by switching to green time for a group of compatible lanes when such a vehicle approaches until it crosses the intersection or by extending green time for such a group of compatible lanes.

\footnotetext{
${ }^{2}$ It is noteworthy to mention here that we assume CAVs communicate with the intersection controller while human-driven vehicles cannot.

${ }^{3}$ Here, we define $T_{t}$ as a constant threshold to separate platoons. However, it is clear that in different traffic instances, the optimal value of $T_{t}$ is different.

${ }^{4}$ Similar to $T_{t}, X_{t}$ can also be optimized in different traffic conditions. However, here we introduced this threshold to highlight the issue of fairness.
} 


\subsection{An example implementation of the PRRP-framework}

First note that for notational convenience, we eliminate the index $i$ to refer to group $i$ of compatible lanes. Also remark that throughout this example, the Negative Exponential distribution is adopted as the distribution of headways. Furthermore, we use the location $L_{p}$ of the last CAV in the queu 5 , as the information of CAVs (i.e., $\left.I_{c}\right)$ received by the intersection controller. Now, to estimate $E\left[N \mid L_{p}=l_{p}\right]$, we have applied the model provided in [22], which estimates the number of queued up vehicles in each lane at the end of the associated red time given the location of the last $\mathrm{CAV}$ in the queue. In this regard, by defining $p$ as the (known) proportion of CAVs, the mentioned model results in the following expected value of the queue length:

$E\left[N \mid L_{p}=l_{p}\right]=\sum_{n=l_{p}}^{\infty} n \cdot \frac{(1-p)^{n} P(N=n)}{\sum_{k=l_{p}}^{\infty}(1-p)^{k} P(N=k)}$.

In Equation (3), $P(N=n)$ refers to the probability that the number of vehicles that have arrived during the corresponding red time equals $n$, without considering any information on the location of CAVs. Under the assumption of a Negative Exponential distribution for the distribution of headways, $P(N=n)$ is $P$ oisson distributed ${ }^{6}$ and equal to

$P(N=n)=\frac{e^{-(\lambda \cdot r)}(\lambda \cdot r)^{n}}{n !}$

In Equation (4), $r$ is the red time and $\lambda$ is the total arrival rate of vehicles (based on the arrival rate of CAVs and their proportion, data of neighboring intersections, or recent history).

Regarding the second term in Equation (2), first, we formulate the probability that the number of vehicles that move with headways shorter than $T_{t}$ joining the queued up vehicles equals $z$ (in other words, the probability that a headway exceeds $T_{t}$ between vehicle number $z$ and $z+1$ for the first time) by

$P(Z=z)=P\left(T_{h_{1}} \leq T_{t}, T_{h_{1,2}} \leq T_{t}, \ldots\right.$,

$\left.T_{h_{z-1, z}} \leq T_{t}, T_{h_{z, z+1}}>T_{t}\right)$

where $T_{h_{1}}$ denotes the headway between the first newly arriving vehicle and the queued vehicles and $T_{h_{i, j}}$ is the headway between vehicles $i$ and $j$. Based on a Negative Exponential distribution, the probability that a headway $T_{h_{i, j}}$ exceeds a defined threshold $\left(T_{t}\right)$ is

$P\left(T_{h_{i, j}}>T_{t}\right)=e^{-T_{t} \lambda}$

Accordingly, by using a Geometric distribution, the formulation presented in Equation (5) can be calculated by

$P(Z=z)=e^{-T_{t} \lambda} \cdot\left(1-e^{-T_{t} \lambda}\right)^{z}$

Second, we use again a Poisson distribution as the probability distribution of the total number of vehicle arrivals during the crossing of the queued up vehicles, as is shown in Equation (8):

$P(V=v)=\frac{e^{-(\lambda \cdot g)}(\lambda \cdot g)^{v}}{v !}$

\footnotetext{
${ }^{5} L_{p}$ is counted as the number of vehicles from the stop line by dividing the location of the last CAV by the average length of vehicles.

${ }^{6}$ We note that, in practice, other distributions could be used as well (for instance based on existing data).
} 
with $g=E\left[N \mid L_{p}=l_{p}\right] / S$.

Now, we can use

$$
E[J(V, Z)]=\sum_{z=0}^{\infty} z \cdot\left[P(Z=z) \cdot \sum_{v=z}^{\infty} P(V=v \mid Z=z)\right]
$$

to calculate the second term in Equation (2). Equation (9) represents the expected value of the number of

vehicles joining the queued up vehicles by considering the probability of two events: (1) the probability that a headway exceeds $T_{t}$ after vehicle number $z$ and (2) the probability that total $v(\geq z)$ vehicles arrive at the intersection during the crossing of the queued up vehicles given that $z$ vehicles are there with short headways.

According to Equations (7) and (8), $Z$ and $V$ are dependent. However, we use

$P(V=v \mid Z=z) \approx P(V=v)$

as the approximation in this example for simplicity 7 Now, by defining $X_{t}$, it becomes possible to estimate the number of vehicles in the platoon (Equation (2)) and, consequently, determine the required green time by Equation (1).

Next, we have implemented the strategy illustrated in Fig. 3 to deal with the prioritization of special vehicles in this example. We assume here that all special vehicles have wireless communication on-board. In this apparatus, since the arrival rate of emergency vehicles is usually low (or even negligible) compared to the total arrival rate of vehicles and, more importantly, due to the nature of this class of vehicles, we give absolute priority to the group of compatible lanes that has an emergency vehicle. Contrary to emergency vehicles, the arrival rate of heavy vehicles is usually considerably larger. Therefore, to decide whether or not to give priority

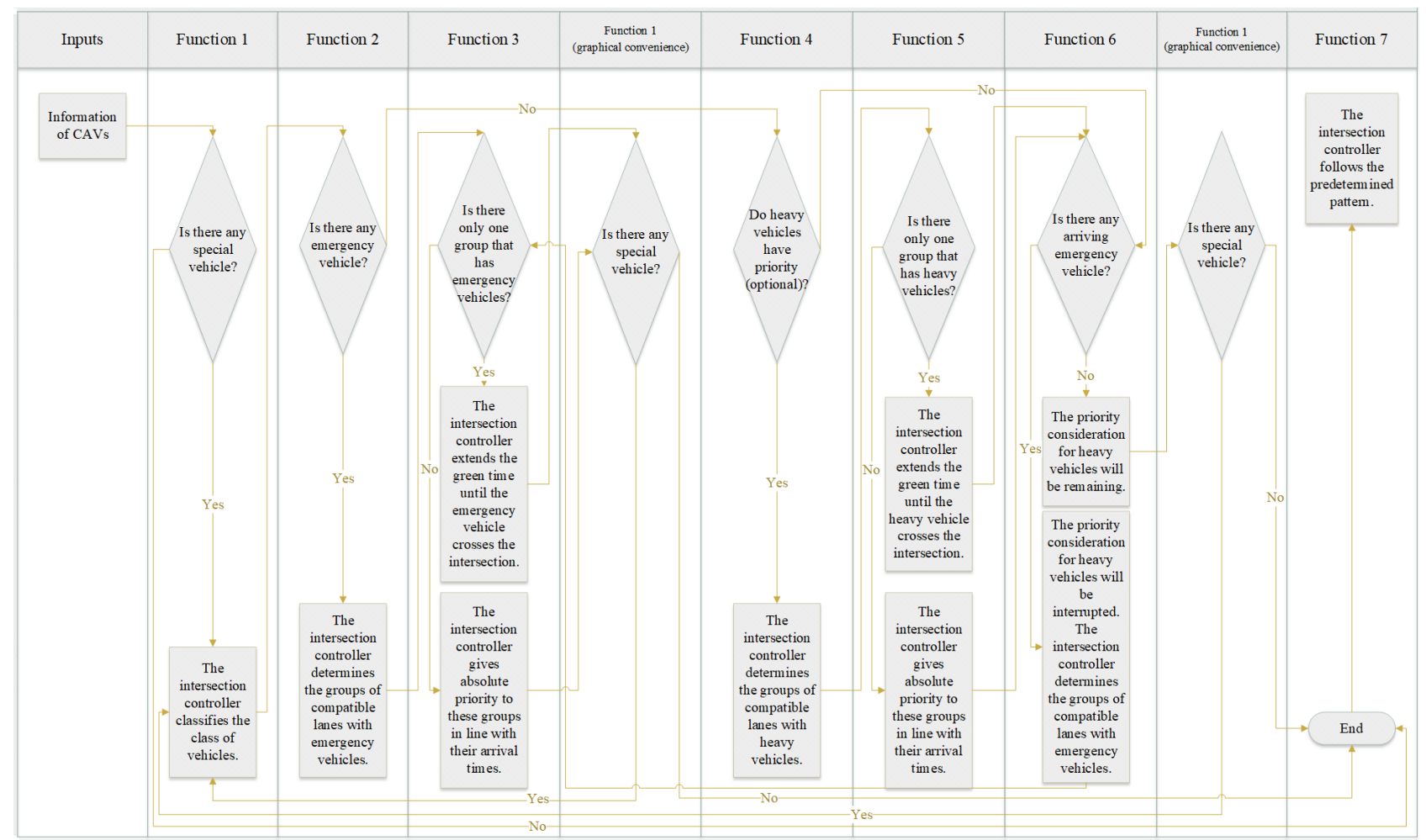

Figure 3: The algorithm for considering different priority treatments for different classes of vehicles

\footnotetext{
${ }^{7}$ Our simulations show that Equation $\sqrt{10}$ is a good approximation, since the corresponding time boundary $(g)$ is relatively small. However, more intricate expressions and/or data calibration could be used as well.
} 
(a)

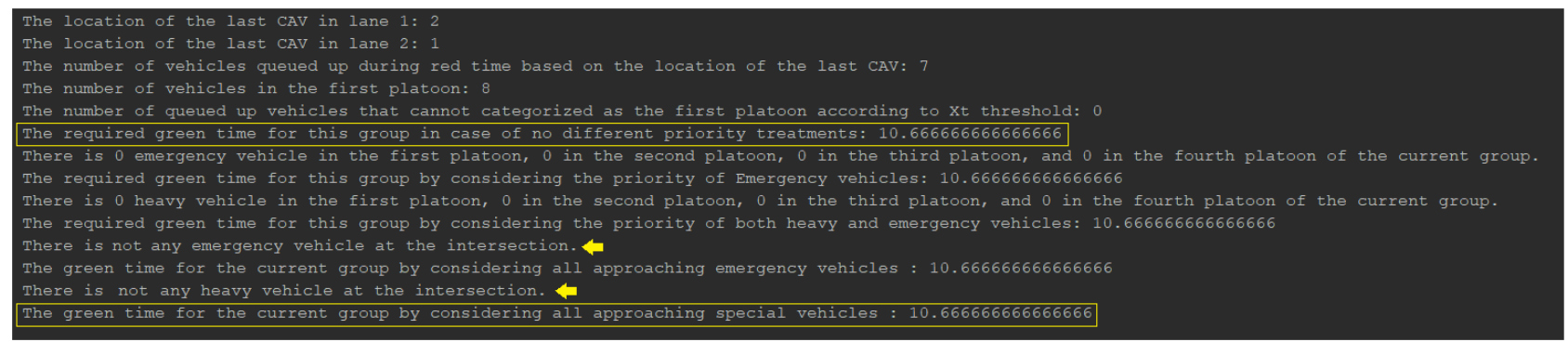

(b)

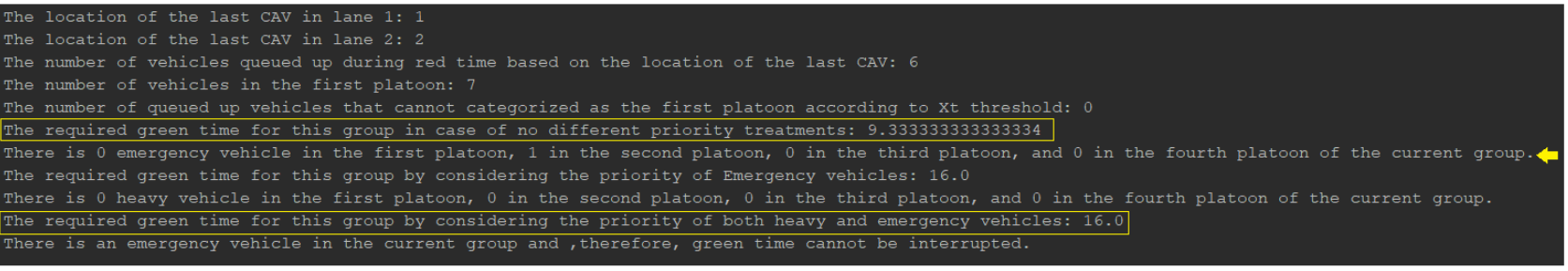

(c)

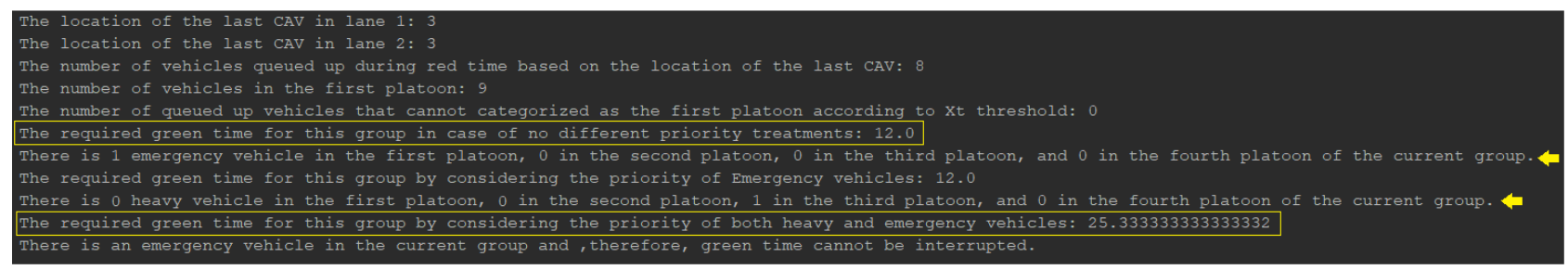

Figure 4: Some green time inferences of the PRRP-framework in different conditions

to these vehicles, we consider the following possible scenarios:

(1) Some heavy vehicles arrive at the intersection in conflicting groups of compatible lanes at the time that green time is extended for the crossing of a previously detected heavy vehicle.

(2) The overall congestion at the intersection is that high that the adverse effect of queued up vehicles is much more severe than that of the stop-and-go events of the detected heavy vehicle.

If either of these scenarios happens, the underlying reasons (in terms of sustainability and economic considerations) for giving priority to a heavy vehicle will be violated. Therefore, by considering the total arrival rate and also the arrival rate of heavy vehicles, we decide on whether to give priority to heavy vehicles or not (Function 4 in Fig. 3).

\section{Operation representation and efficiency evaluation of the PRRP-framework}

To represent the operation of the PRRP-framework, first, we develop Python code to show the (effective) green time inferences of this framework for a group of compatible lanes in different conditions using our showcase equations. To do this, threshold $T_{t}$ and threshold $X_{t}$ are assumed 4 seconds and 20 vehicles, respectively. Moreover, we assume that the intersection controller gives priority to heavy vehicles. Furthermore, the average service rate of vehicles and the total arrival rate are considered to be 2700 and 2400 (vehs/h) (distributed evenly among all lanes). Finally, we consider 25\%,1\%, and 4\%, as the proportion of CAVs, emergency vehicles, and heavy vehicles (respectively, without considering the overlaps) in the traffic composition. Some of the inferences are provided in Fig. 4 . 
In this regard, Fig. 4 (a) is related to the condition in which no special vehicle is at the intersection and, therefore, the duration of green time is merely the result of the number of vehicles in the composed platoon. Fig. 4 (b) shows the operation of this framework when there is an emergency vehicle in the progressing group. Finally, Fig. 4 (c) investigates how the intersection controller reacts in case of both emergency and heavy vehicles 8

We have also used an internal script ${ }^{9}$ to simulate the green time inferences of the PRRP-framework in VISSIM. Using the VISSIM model, we apply the same traffic composition in a general intersection with four groups of compatible lanes (Fig. 1) to compare the efficiency of the PRRP-framework and traditional approaches of managing intersections, namely pre-timed and actuated signals, in terms of average delay. In this comparison:

(1) the thresholds of the PRRP-framework and the average service rate are similar to what we have assumed in representing the green time inferences.

(2) 20-meter extension loops (to detect if there is a need for green time extensions) are used in each specific lane to form actuated signals with a minimum (maximum) green time equal to 7 seconds (25 seconds)

(3) we use the Webster method (see [23]) to determine the optimal cycle time for pre-timed signals ${ }^{11}$

We use different arrival rates to compare the above-mentioned mechanisms in different saturation degrees. In this regard, 10 series of (one-hour) simulations have been carried out for each specific mechanism with considering total arrival rates equal to 800, 1600, and 2400 (vehs $/ h$ ) (which represent under-saturated, moderate-saturated, and near-saturated conditions, respectively). The average results of simulation tests are shown in Fig. 5

As Fig. 5 demonstrates, comparing with the optimal pre-timed signal setting, the PRRP-framework can help improve the performance of an intersection in terms of average delay. The main reason behind this improvement is that the stochasticity of arrivals will be accounted for spontaneously by the PRRP-framework. It seems that the improvement is greater for higher arrival rates.

Indeed, at low arrival rates (arrival rate $=800(v e h s / h)$ in Fig. 5), it is expected to have cycles without overflow queues. However, when signals operate close to the state of full saturation (arrival rate $=2400(v e h s / h)$ in Fig. 5), the probability that some cycles have overflow queues (with consequently higher average delay) will also increase. Accordingly, the mechanism of the PRRP-framework in treating platoons of vehicles becomes more influential in the case of near-saturated conditions.

Comparing with the defined actuated mechanism, we can see that our proposed framework is competitive to the actuated signals which require a considerable amount of detectors. Secondly, it must be stressed that the proposed algorithm for considering different priority treatments renders the PRRP-framework capable of prioritizing special vehicles over others while both the pre-timed and actuated signals treat all vehicles uniformly.

Regarding the priority setting, the green time duration in the PRRP-framework is adjusted to minimize the stopping time of special vehicles (in some cases) at the cost of the average delay of ordinary vehicles. To assess this further, we have repeated the VISSIM simulation for alternative proportions of special vehicles while considering a constant total arrival rate equal to 2400 (vehs/h). The results are shown in Fig. 6

According to Fig. 6, if the proportion of defined special vehicles in the traffic composition is low (or, for

\footnotetext{
${ }^{8}$ One can also see the operation of the intersection controller in case of both emergency and heavy vehicles in conflicting groups by using the source code available at https://github.com/HossseinMoradi/Project1.

${ }^{9}$ https://github.com/HossseinMoradi/Project2

${ }^{10}$ https://github.com/HossseinMoradi/Project3

${ }^{11}$ We also distribute arrivals equally among approaching links, to help pre-timed signals to have their best performance.
} 


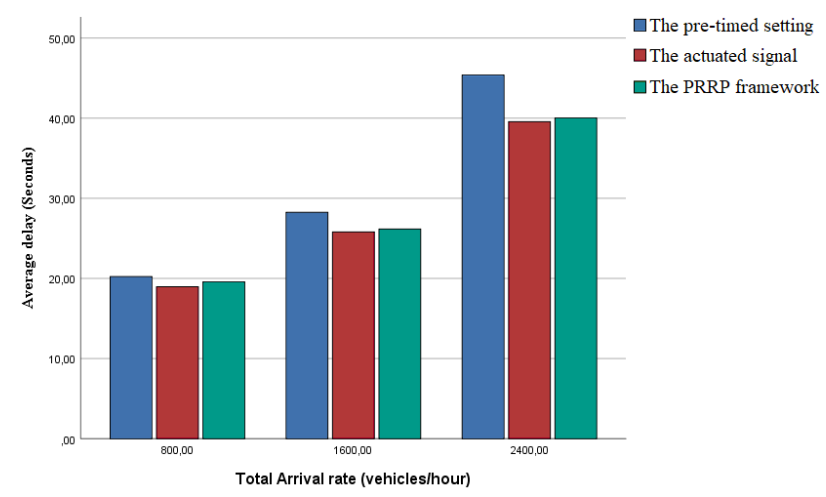

Figure 5: Comparing the average delay of the pre-timed setting, the actuated mechanism, and the PRRP-framework in different arrival rates

example, only emergency vehicles get priority), we can see that the PRRP-framework outperforms not only the pre-timed setting but also the actuated mechanism. On the contrary, when the arrival rate of special vehicles grows, the average delay of the PRRP-framework becomes higher than that of the actuated mechanism. This increase in the average delay has its origin in the stochastic arrival of special vehicles which entails the variation of green (red) time irrespective of current demands. This result clearly highlights the importance of flexibility in deciding whether or not to give priority to some special vehicles in different conditions (Function 4 in Fig. 33), which is left for future research.

Based on the obtained results, we see that the proposed PRRP-framework can gradually improve pre-timed signals by (1) using the communication abilities of CAVs and (2) employing the potential of probability theory in addressing the inherent uncertainty of traffic flows (even with a low proportion of CAVs) to efficiently manage mixed traffic. Furthermore, by using the RR algorithm in this framework, which treats platoons of vehicles as units and by also embedding a prioritizing algorithm, the PRRP-framework can address demand fluctuations naturally and give priority to special vehicles as well.

\section{Integration of the PRRP-framework into a speed advisory system}

Speed advisory systems have been developed based on emission estimation models to determine the optimal speed-trajectory profile for (connected) vehicles to minimize energy consumption, and consequently, emissions 24]. In this section, we employ the potentials of wireless communications to connect the PRRP-framework with

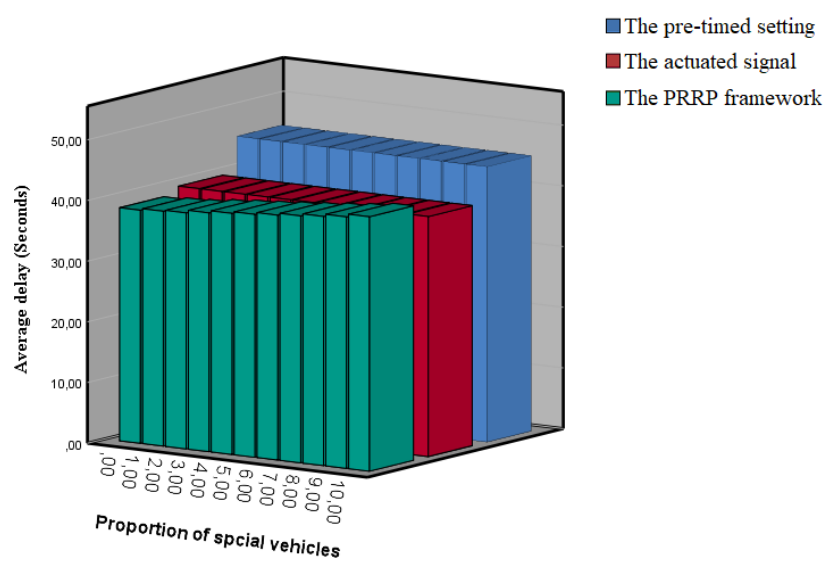

Figure 6: Variations of average delay with considering different proportions (in percent) of special vehicles 
a test case speed advisory system to study the integration effects of the proposed intersection control method and such a control-related strategy.

Regarding the test case speed advisory system, we customize the model employed in [25] with the following modifications:

(1) The original model has been designed to determine the optimal speed of the leading vehicle in each platoon. However, delivering the optimal speed to a human driver (if the leading vehicle is not a connected one) does not seem to be feasible. At the same time, based on the results of previous studies, it is expected that using speed advisory systems for CAVs not only helps them reduce their own energy consumption, but also assists in improving the fuel economy of human-driven vehicles due to the car-following relationship [26]. Given this explanation, we choose to apply this speed advisory system for CAVs, whether or not they are platoon leaders.

(2) The unknown condition of approaching conventional vehicles is another factor that might change the optimal solution. In other words, the obtained speed-trajectory profiles for CAVs are (probably) not the optimal ones if we process the information of both CAVs and conventional vehicles. ${ }^{12}$ Since we assume that conventional vehicles cannot communicate (whether with the intersection controller or CAVs), we allow CAVs to be driven with the desired speed (according to the defined speed limit) when the corresponding signal turns green. By doing so, CAVs will not be the traffic barriers for conventional vehicles (driving behind them) that can cross the intersection within the same cycle.

(3) The queue dynamics at intersections is another factor that should be taken into account [27]. Here, we assume a constant queue dissipation time.

The following sections explain the applied speed advisory system, which is coupled to the PRRP-framework to form the integrated framework.

\subsection{Energy consumption of $C A V s$}

In this section, we calculate the energy consumption of CAVs by considering the operational differences between gasoline and electric vehicles. First, regarding gasoline vehicles, we use

$$
\begin{aligned}
& F(v(t), a(t))= \\
& \max \left\{\left(\alpha+\beta_{1} \cdot v(t) \cdot R(t)+\left[\beta_{2} \cdot M \cdot v(t) \cdot a^{2}(t)\right]_{a(t)>0}\right), \alpha\right\}
\end{aligned}
$$

which is presented in [28] for estimating the gasoline consumption. In Equation [11], $M, \alpha, \beta_{1}$, and $\beta_{2}$ are the parameters that stand for vehicle weight $(\mathrm{kg})$, idling gasoline consumption rate $(\mathrm{ml} / \mathrm{s})$, efficiency of engine $(m l / k J)$, and efficiency of engine in terms of making accelerations $\left(m l /\left(k J m / s^{2}\right)\right)$, respectively. Furthermore, $a(t)$ and $v(t)$ denote the acceleration $\left(\mathrm{m} / \mathrm{s}^{2}\right)$ and speed $(\mathrm{m} / \mathrm{s})$ of the vehicle at time $t$. Finally, $R(t)$ is the required tractive force $(k N)$, which is given by

$$
R(t)=b_{1}+b_{2} \cdot v^{2}(t)+M \cdot a(t)+M \cdot g \cdot \theta
$$

In Equation (12), $b_{1}$ and $b_{2}$ are the rolling $(k N)$ and aerodynamic resistance $(\mathrm{kg} / \mathrm{m})$. Moreover, $g$ is the gravitational acceleration $\left(\mathrm{m} / \mathrm{s}^{2}\right)$ and $\theta$ is the road gradient.

\footnotetext{
${ }^{12}$ As a case in point, it may cause conventional vehicles driving behind CAVs to get stuck behind a red light.
} 
Second, regarding electric vehicles, the formulation presented in [29] has been adopted for the estimation of the power consumption, as is shown in Equation (13):

$$
\begin{aligned}
P(v(t), a(t))= & \frac{r \cdot \gamma^{2}}{K^{2}}\left(M \cdot a(t)+k \cdot v^{2}(t)+f_{r l} \cdot M \cdot g+\right. \\
& M \cdot g \cdot \theta)^{2}+v(t) \cdot\left(k \cdot v^{2}(t)+f_{r l} \cdot M \cdot g\right. \\
& +M \cdot g \cdot \theta)+\eta \cdot M \cdot a(t) \cdot v(t)
\end{aligned}
$$

In Equation 13, $r$ represents the motor resistance $(\Omega), \gamma$ is the radius of tires $(m)$, and $K$ is a defined factor $(\sqrt{\Omega . m . s})$ that is obtained by multiplying armature constant and magnetic flux. In addition, $k$ and $f_{r l}$ serve as the aerodynamic resistance $(\mathrm{kg} / \mathrm{m})$ and the rolling resistance coefficient, respectively. Finally, it should be considered that electric vehicles are capable of regenerating electricity during decelerating. The efficiency of this process is denoted by $\eta$.

Third, as a way to assimilate Equations (11) and (13), which result in different units $(\mathrm{ml} / \mathrm{s}$ and $W$, respectively), the applied pattern proposes to convert the energy of gasoline into electricity 13 , As a result, we can represent the total energy consumption of CAVs by $E(v(t), a(t))$, whose value is a function of vehicle's speed and acceleration.

\subsection{The formulation of the speed advisory system}

As indicated earlier, the speed advisory system is applied to advise each CAV upon coming into the area of the intersection on a speed profile that is optimized with respect to energy consumption. The corresponding optimization problem is formulated below. Equation $(14)$ is the objective function, which seeks to minimize the total energy consumption $(\mathcal{E})$ for a given number of $J$ CAVs through specifying their optimal speed $v_{1}^{*}, \ldots, v_{J}^{*}$. This optimization problem should be solved subject to the enumerated constraints:

$\min _{v_{1}^{*}, \ldots, v_{J}^{*}} \mathcal{E}=\sum_{j=1}^{J} \int_{t=0}^{t=t_{f_{j}}} E_{j}\left(v_{j}(t), a_{j}(t)\right) d t$

s.t.

$$
\begin{array}{ll}
\text { 1. } & \underline{\mathrm{T}}_{f_{i}} \leq t_{f_{1}}<\ldots<t_{f_{J_{i}}}<\bar{T}_{f_{i}}, \\
\text { 2. } & x\left(t_{f_{j}}\right)=D_{j}, \\
\text { 3. } & 0<v_{j}^{*}<\bar{v} \\
\text { 4. } & \underline{a}_{j} \leq a_{j}^{*} \leq \bar{a}_{j} .
\end{array}
$$

In Equation (14), $E_{j}\left(v_{j}(t), a_{j}(t)\right)$ denotes the energy consumption of $\operatorname{CAV} j(j=1, \ldots, J)$, whether it is an electric or a gasoline vehicle. For $\operatorname{CAV} j$, we have a distinct time interval starting from $t=0$ when CAV $j$ is identified and ending with $t=t_{f_{j}}$ at which this CAV arrives at the intersection.

As the first step to finding the emission-optimal speed profile for CAVs, the intersection controller takes the current intersection phase into account. It should be understood that we want respective $J_{i}$ CAVs (in group $i$ of compatible lanes) to arrive at the intersection at respective times $t_{f_{1}}, \ldots, t_{f_{J_{i}}}$ when the signal is green for group $i$. Therefore, we impose the first constraint where $\underline{\mathrm{T}}_{f_{i}}$ is the lower time boundary and $\bar{T}_{f_{i}}$ is the upper time boundary of the intended green phase.

\footnotetext{
${ }^{13}$ Based on [30, the energy of one gallon of gasoline is approximately equal to $33.7 k W h$ electricity. As a result, we consider 10 as the converting coefficient.
} 
As the second step, the intersection controller retrieves the current speed and position of $\operatorname{CAV} j$. Accordingly, considering the current position of $\mathrm{CAV} j$, if its distance to the intersection at time $t=0$ equals $D_{j}$, then the relative position of this $\mathrm{CAV}$ at time $t=t_{f_{j}}$ (i.e., $x\left(t_{f_{j}}\right)$ ) is $D_{j}$ as compared to its initial position.

By using the above-mentioned steps, we can derive the possible speed profiles for $\operatorname{CAV} j$, in the defined time

(3) Simulation of the integrated framework ${ }^{14}$. We have incorporated the speed advisory system in the PRRPframework explained in Section 2 .

To evaluate the performance of the integrated framework, we utilize a general four-link intersection (with the same layout as we used before) and consider the total arrival rate to be equal to 2400 (vehs $/ h)^{15}$ to add the results of:

\footnotetext{
${ }^{14}$ https://github.com/HossseinMoradi/Project4

${ }^{15}$ Since this type of signals are meant to operate as closely as possible to the state of full saturation, the results of this arrival rate (in our showcase) are of the utmost importance.
} 


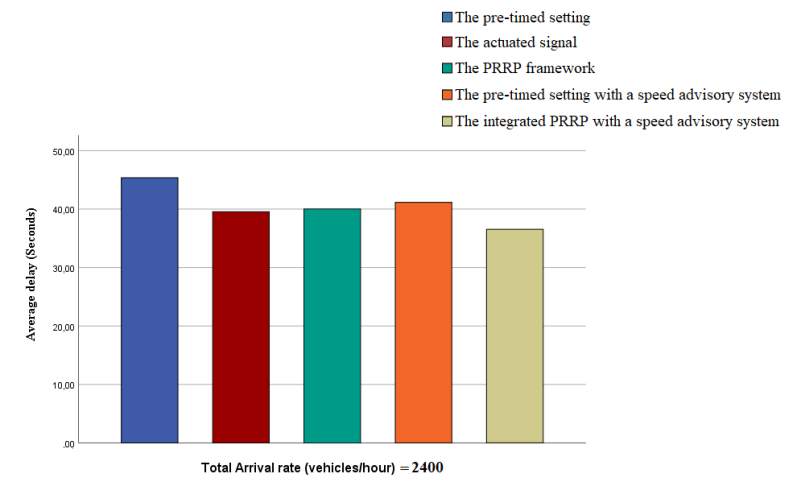

Figure 7: Comparing the average delay of the pre-timed setting, the actuated mechanism, the $P R R P$-framework, the speed advisory system, and the integrated framework in a near saturated condition

- using a pre-timed signal setting as the signal control method along with applying the speed advisory system

- using both the PRRP-framework and the explained speed advisory system (the integrated framework)

to the picture. In this comparison, we also use the same traffic composition as in Section 3 . Fig. 7 compares the obtained average delays.

According to Fig. 7, while applying only the speed advisory system can help improve the performance of an intersection in dealing with the issue of average delay, the integration of the speed advisory system into the PRRP-framework (labeled as the integrated framework) outperforms all the mechanisms by resulting in a lower average delay. As a case in point, compared to the developed pre-timed signal setting (in its best scenario), using the integrated framework leads to a $19.5 \%$ reduction in the average delay, which is more than respectively $13 \%, 12 \%$, and $10 \%$ reductions obtained from the actuated signal, the PRRP-framework, and the speed advisory system.

The most reason for the observed improvement in the integrated framework is the complementary strengths brought by the PRRP-framework and by the speed advisory system. On the one hand, as is shown in Section 3, the proposed PRRP-framework determines the proportional green time by aligning green time duration to the corresponding demands. On the other hand, the speed advisory system helps traffic fleets, in general, and CAVs, in particular, to better use that time. In this case, when the signal is red, CAVs drive with the optimal speed which helps them to arrive at the intersection during green time durations without the lost time needed for re-acceleration from a stop condition.

A series of simulations has also been carried out comparing the efficiency of the mentioned mechanisms in terms of emissions reduction. Accordingly, Fig. 8 presents the obtained results for the total $C O$ emissions $(g)$ at the intersection with considering total arrival rate equal to 2400 (vehs/h).

According to Fig. 8 , the results show that by considering the optimal pre-timed setting as the benchmark:

- the actuated mechanism results in a $3.77 \%$ reduction in $C O$ emissions mainly due to its ability in extending green time;

- the PRRP-framework leads to a $4.77 \%$ reduction in $C O$ emissions. In this case, we can see that while the average delay of the PRRP-framework is (slightly) higher than that of the actuated mechanism, it works better in terms of emissions reduction due to its capability for considering priority treatments for heavy vehicles; 
- the speed advisory system reduces emissions by $5.16 \%$ through determining the optimal speed of approaching CAVs;

- the integrated framework brings a $9.9 \%$ reduction in $C O$ emissions that results from (1) reducing the number of stop-and-go events, (2) reducing the average delay, (3) moving CAVs with the optimal speed, and (4) giving priority to heavy vehicles. In each specific condition, one of the above-mentioned reasons (whose existence depends on whether the interaction of incorporated systems or their separate functions) becomes dominant and helps the integrated framework to have superior performance.

\section{Conclusion and future research directions}

This paper has first proposed the PRRP-framework to address the need for a rule-based model capable of managing mixed traffic (with considering the concomitant complexities) and has secondly presented an integrated framework (built upon the PRRP-framework and a speed advisory system) with even better performance.

Regarding the PRRP-framework, our simulations first showed how this framework infers green time durations on the grounds of the current demand and the presence of special vehicles. Then the relative efficiency of the PRRP-framework has been confirmed, compared to the traditional signals.

Regarding the integrated framework, the simulations showed that the proposed framework makes contributions with regard to the reduction of emissions whose amount is higher than that for (1) the optimal pre-timed signal, (2) the actuated mechanism, (3) the speed advisory system, and (4) the PRRP-framework. It was also shown that the integrated framework works better than the enumerated ones in terms of the average delay.

It should be noted that the implementation of the integrated framework is an illustration of a key rationale behind this work: integration among solutions is a prerequisite to reach sustainable results. With this momentum, future research studies can be developed to integrate other solutions into this framework. As a case in point, a rigorous analysis of the effects of embedding signal synchronization systems into this framework is an interesting topic left for future work.

\section{References}

[1] X. Qian, F. Altché, J. Grégoire, and A. de La Fortelle, Autonomous intersection management systems: criteria, implementation and evaluation. IET Intelligent Transport Systems 11 (2017) 182-189.

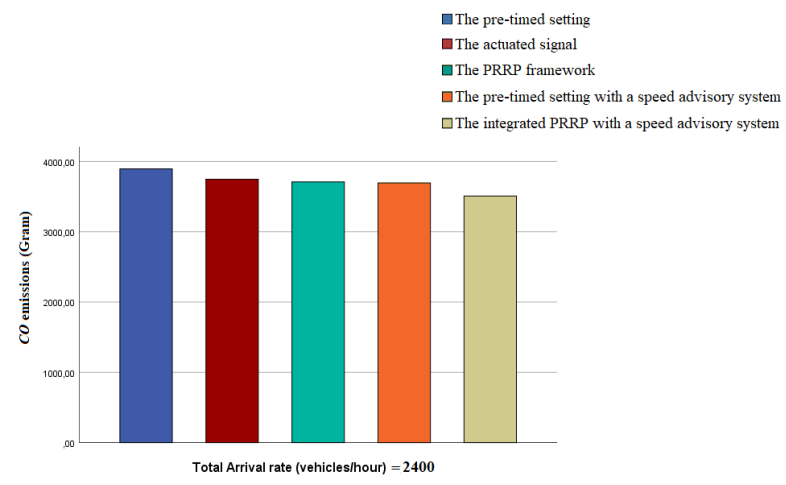

Figure 8: Comparing $C O$ emissions resulting from the use of the pre-timed setting, the actuated mechanism, the PRRP-framework, the speed advisory system, and the integrated framework in a near saturated condition 
[2] K. Dresner and P. Stone, A multiagent approach to autonomous intersection management. Journal of artificial intelligence research 31 (2008) 591-656.

[3] G. Sharon and P. Stone, A protocol for mixed autonomous and human-operated vehicles at intersections. International Conference on Autonomous Agents and Multiagent Systems, Springer, (2017) 151-167.

[4] R. Blokpoel and W. Niebel, Advantage of cooperative traffic light control algorithms. IET Intelligent Transport Systems 11 (2017) 379-386.

[5] H. Moradi, M.H. Sebt, and E. Shakeri, Evaluating the impacts of virtual organization absorption on the quality of urban private constructions; the system dynamics approach. Systemic Practice and Action Research 39 (2019) 443-462.

[6] Y. Feng, K.L. Head, S. Khoshmagham, and M. Zamanipour, A real-time adaptive signal control in a connected vehicle environment. Transportation Research Part C: Emerging Technologies 55 (2015) 460-473.

[7] R. Blokpoel, S. Hausberger, and D. Krajzewicz, Emission optimised control and speed limit for isolated intersections. IET Intelligent Transport Systems 11 (2017) 174-181.

[8] A.D. Bachelder and C.F. Foster, Emergency vehicle traffic signal preemption system. Google Patents 7 (2008) 327-280.

[9] O. De Keyser, M. Hillewaere, P. Audenaert, and B. Maenhout, Optimising the public transport priority at road intersections. IET Intelligent Transport Systems 12 (2018) 986-994.

[10] J. Walraevens, T. Maertens, P. Audenaert, and S. Wittevrongel, Giving priority access to freight vehicles at signalized intersections. In the 32nd Annual Conference of the Belgian Operational Research Society (Orbel 32) (2018) 101-102.

[11] Q. He, K. L. Head, and J. Ding, Multi-modal traffic signal control with priority, signal actuation and coordination. Transportation Research Part C: Emerging Technologies 46 (2014) 65-82.

[12] L. Li and F.-Y. Wang, Cooperative driving at blind crossings using intervehicle communication. IEEE Transactions on Vehicular Technology 55 (2006) 1712-1724.

[13] P. Li and X. Zhou, Recasting and optimizing intersection automation as a connected-and-automated-vehicle $(C A V)$ scheduling problem: A sequential branch-and-bound search approach in phase-time-traffic hypernetwork. Transportation Research Part B: Methodological 105 (2017) 479-506.

[14] J. Walraevens, B. Steyaert, M. Moeneclaey, and H. Bruneel Delay analysis of a HOL priority queue. Telecommunication Systems 30 (2005) 81-98.

[15] S. Wittevrongel, S. De Vuyst, C. Sys, and H. Bruneel, A reservation-based scheduling mechanism for fair QoS provisioning in packet-based networks. 26th International of Teletraffic Congress (2014) 1-8.

[16] H. Moradi, M. H. Sebt, and E. Shakeri, Toward improving the quality compliance checking of urban private constructions in Iran: An ontological approach. Sustainable Cities and Society 38 (2018) 137-144. 
[17] A. Stevanovic, M. J. Stevanovic, K. Zhang, and S. Batterman, Optimizing traffic control to reduce fuel

[18] D. Krajzewicz, J. Erdmann, M. Behrisch, and L. Bieker, Recent development and applications of SUMOSimulation of Urban MObility. International Journal On Advances in Systems and Measurements 5 (2012).

[19] J. Lioris, R. Pedarsani, F. Y. Tascikaraoglu, and P. Varaiya, Platoons of connected vehicles can double throughput in urban roads. Transportation Research Part C: Emerging Technologies 77 (2017) 292-305.

[20] K. Y. Liang, J. Mårtensson, and K. H. Johansson, Heavy-duty vehicle platoon formation for fuel efficiency. IEEE Transactions on Intelligent Transportation Systems 17 (2016) 1051-1061.

[21] X. J. Liang, S. I. Guler, and V. V. Gayah, Signal timing optimization with connected vehicle technology: Platooning to improve computational efficiency. Transportation Research Record 2672 (2018) 81-92.

[22] G. Comert and M. Cetin, Queue length estimation from probe vehicle location and the impacts of sample size. European Journal of Operational Research 197 (2009) 196-202.

[23] H. Taale, Comparing methods to optimise vehicle actuated signal control. Eleventh International Conference on Road Transport Information and Control (2002) 114-119.

[24] M. Barth and K. Boriboonsomsin, Energy and emissions impacts of a freeway-based dynamic eco-driving system. Transportation Research Part D: Transport and Environment 14 (2009) 400-410.

[25] X. He and X. Wu, Eco-driving advisory strategies for a platoon of mixed gasoline and electric vehicles in a connected vehicle system. Transportation Research Part D: Transport and Environment 63 (2018) 907-922.

[26] N. Wan, A. Vahidi, and L. Luckow, Optimal speed advisory for connected vehicles in arterial roads and the impact on mixed traffic. Transportation Research Part C: Emerging Technologies 69 (2016) 548-563.

[27] X. He, H. X. Liu, and X. Liu, Optimal vehicle speed trajectory on a signalized arterial with consideration of queue. Transportation Research Part C: Emerging Technologies 61 (2015) 106-120.

[28] D. Biggs and R. Akcelik, Energy-related model of instantaneous fuel consumption. Traffic Engineering and Control 27 (1986) 320-325.

[29] X. Wu, D. Freese, A. Cabrera, and W.A. Kitch Electric vehicles' energy consumption measurement and estimation. Transportation Research Part D: Transport and Environment 34 (2015) 52-67.

[30] M. EPA New fuel economy and environment labels for a new generation of vehicles. Regulatory Announcement EPA-420-F-11-017, US Environmental Protection Agency (2011). 